\title{
Radiative production of nonthermal dark matter
}

\author{
Kunio Kaneta, ${ }^{1, *}$ Yann Mambrini, ${ }^{2, \dagger}$ and Keith A. Olive ${ }^{1, \$}$ \\ ${ }^{1}$ William I. Fine Theoretical Physics Institute, School of Physics and Astronomy, \\ University of Minnesota, Minneapolis, Minnesota 55455, USA \\ ${ }^{2}$ Laboratoire de Physique Théorique (UMR8627), CNRS, Univ. Paris-Sud, \\ Université Paris-Saclay, 91405 Orsay, France
}

(Received 18 January 2019; published 12 March 2019)

\begin{abstract}
We compare dark matter production from the thermal bath in the early universe with its direct production through the decay of the inflaton. We show that even if dark matter does not possess a direct coupling with the inflaton, Standard Model loop processes may be sufficient to generate the correct relic abundance.
\end{abstract}

DOI: 10.1103/PhysRevD.99.063508

\section{INTRODUCTION}

Despite indirect but clear evidence [1] of the presence of a large amount of dark matter in our Universe, its nature still remains elusive. The absence of any signal in direct detection experiments XENON [2], LUX [3] and PandaX [4] question the weakly coupled dark matter paradigm. Simple extensions of the Standard Model such as the Higgs-portal [5,6], Z-portal [7], or even $Z^{\prime}$-portal [8] as well as more complex extensions such the minimal supersymmetric Standard Model [9-11] have a large part of their parameter space (if not all) excluded when combining direct, indirect and accelerator searches (for a review on weakly interacting massive particles (WIMP) searches and models, see [12]). As a consequence, it is useful to look for different scenarios, including those with ultraweak couplings [13] (see [14] for a review), or the possibility that dark matter production occurred at very early stages of reheating after inflation as in the case of gravitino production $[11,15,16]$.

In this context, it has been confirmed that dark matter production is naturally feasible in a wide variety of models such as $\mathrm{SO}(10)$ grand unification [17], anomaly free U(1)' models [18], spin-2 portal [19] high scale supergravity [20-24] or moduli portal [25]. In all these models, it has been shown that effects of noninstantaneous reheating [26,27] and noninstantaneous thermalization [28] are non-negligible.

\footnotetext{
*kkaneta@umn.edu

†ann.mambrini@th.u-psud.fr

olive@umn.edu
}

Published by the American Physical Society under the terms of the Creative Commons Attribution 4.0 International license. Further distribution of this work must maintain attribution to the author(s) and the published article's title, journal citation, and DOI. Funded by SCOAP .
As in the case of gravitino production during inflationary reheating, in many models, dark matter is produced from the annihilation of thermal Standard Model particles, and one neglects the direct production of dark matter $\chi$ from the decay of the inflaton, $\phi$. It was shown $[21,22,29,30]$ that the branching ratio $\operatorname{BR}(\phi \rightarrow \chi \chi)$, is constrained to be very small (below $10^{-8}$ for a $100 \mathrm{GeV}$ dark matter and a reheating temperature $T_{R H} \gtrsim 10^{10} \mathrm{GeV}$ ). In this paper, we compare the dark matter production rate from both the thermal bath and direct decay. We show that if dark matter is produced from the thermal bath, independent of the tree level branching ratio of inflaton decay to dark matter, one cannot ignore the radiative decay of the inflaton into dark matter. We further show that the radiative decay may well dominate the production rate thus providing the main source for relic abundance in the Universe. As a particular example, we also consider the radiative contribution for gravitino production in models of high scale supersymmetry.

The paper is organized as follows. We first compute the dark matter abundance is Sec. II, then apply it to a generic microscopic model in Sec. III before looking to consequences in supersymmetric scenarios in Sec. IV. We conclude in Sec. V.

\section{DARK MATTER PRODUCTION}

\section{A. Generalities}

We presume that the dark matter is not produced in thermal equilibrium during reheating and as a consequence, the dark matter abundance, $n_{\chi}$, in the early universe is much lower than the thermal density $n_{\gamma}$. If we further assume that the dark matter annihilation process, $n_{\chi} n_{\chi} \rightarrow n_{\gamma} n_{\gamma}$, is also out of equilibrium, since $n_{\chi}^{2} \ll n_{\gamma}^{2}$, we can write the Boltzmann equation as

$$
\frac{d n_{\chi}}{d t}+3 H n_{\chi}=R(T)
$$


with

$R(T)=\int f_{1} f_{2} \frac{E_{1} E_{2} d E_{1} d E_{2} d \cos \theta_{12}}{1024 \pi^{6}} \int\left|\mathcal{M}_{f i}\right|^{2} d \Omega_{13}$,

for a process $12 \rightarrow 34$ with 1 and 2 representing Standard Model particles in the thermal bath and 3, 4 representing the dark matter candidate, with $f_{1}$ and $f_{2}$ being the thermal distribution functions of the incoming particles 1 and 2 and $d \Omega_{13}$ is the solid angle between the particle 1 and 3 in the center of mass frame.

To compute the relic abundance, the strategy is straightforward. To solve Eq. (1), one needs to know the relation between $T$ and the cosmological scale parameter, $a$. We can then use the Hubble parameter $H=\frac{\dot{a}}{a}$ to express $t$ as function of $T$ before integrating Eq. (1). The dependence of $T$ on $a$ is obvious in a pure radiation dominated or a pure matter dominated era $\left(T \propto a^{-1}\right)$ due to entropy conservation. However, during reheating, the temperature grows from effectively 0 (when the Universe is still dominated by inflaton oscillations) to a maximum temperature, $T_{\max }$, [26,27]. Subsequently, the temperature decreases to $T_{\mathrm{RH}}$ when the inflaton is (nearly) fully decayed. The maximum temperature is determined by the inflaton decay width, $\Gamma_{\phi}$ and is approximated by $T_{\max } \simeq 0.5\left(M_{\phi} / \Gamma_{\phi}\right)^{1 / 4} T_{\mathrm{RH}}$, where $M_{\phi}$ is the inflaton mass. During this period, the density of Universe is a mixture between the inflaton and the growing radiation density. It has been shown in $[26,27]$ that solving the set of conservation of energy conditions for the inflaton density $\rho_{\phi}$, the radiation density $\rho_{R}$ combined with the Friedmann equation:

$$
\begin{aligned}
& \frac{d \rho_{\phi}}{d t}+3 H \rho_{\phi}=-\Gamma_{\phi} \rho_{\phi} \\
& \frac{d \rho_{R}}{d t}+4 H \rho_{R}=\Gamma_{\phi} \rho_{\phi} . \\
& H^{2}=\frac{\rho_{\phi}}{3 M_{P}^{2}}+\frac{\rho_{R}}{3 M_{P}^{2}},
\end{aligned}
$$

where $\Gamma_{\phi}$ is the width of the inflaton, ${ }^{1}$ and $M_{P}$ the reduced Planck mass ${ }^{2}$ one obtains

$$
T \propto a^{-3 / 8},
$$

as $T$ decreases from $T_{\max }$ to $T_{\mathrm{RH}}$. Defining the comoving yield $Y$

\footnotetext{
${ }^{1} \mathrm{~A}$ more precise computation should distinguish $\Gamma_{\phi}$ into the part contributing to the thermal bath $\Gamma_{\phi}^{\gamma}$ and the part contributing to the dark sector $\Gamma_{\phi}^{\chi}$. However, we will always consider (and justify) $\Gamma_{\phi}^{\chi} \ll \Gamma_{\phi}^{\gamma}$ throughout our study.$$
{ }^{2} M_{P}=\frac{1}{\sqrt{8 \pi}} M_{\mathrm{Pl}}=2.4 \times 10^{18} \mathrm{GeV} .
$$

$$
Y=\frac{n}{T^{8}},
$$

the Eq. (1) becomes

$$
\frac{d Y}{d T}=-\frac{8}{3} \frac{R(T)}{H T^{9}}
$$

If we further make the approximation that the inflaton dominates the total energy density between $T_{\max }$ and $T_{\mathrm{RH}}$, we can express the Hubble parameter in terms of $\rho_{\phi}$ which falls as $a^{-3} \sim T^{8}$ with a constant of proportionality depending on the inflaton decay rate,

$$
H(T) \approx \frac{5}{6} \frac{\alpha}{\Gamma_{\phi} M_{P}^{2}} T^{4} .
$$

In Eq. (7), $\alpha=\frac{g(T) \pi^{2}}{30}$, where $g(T)$ counts the relativistic degrees of freedom of the thermal bath at the temperature $T$ $\left(g_{*}=106.75\right.$ in the Standard Model) so that $\rho_{R}=\alpha T^{4}$. In this case, Eq. (6) becomes

$$
\frac{d Y}{d T}=-\frac{24 c}{5 \sqrt{3 \alpha}} \frac{R(T) M_{P} T_{R H}^{2}}{T^{13}}
$$

where we have taken

$$
\frac{3}{2} c H\left(T_{\mathrm{RH}}\right)=\frac{3}{2} c \sqrt{\frac{\alpha}{3}} \frac{T_{\mathrm{RH}}^{2}}{M_{P}}=\Gamma_{\phi}
$$

to define the reheating temperature, where $c \approx 1.2$ is a constant obtained from a numerical integration [30,31]. The particular choice of a dark matter candidate and its interactions will determine $R(T)$ and allow for the integration of Eq. (8).

\section{B. Dark matter production from the thermal bath}

It has been shown in [17] that dark matter can be produced in the very early stages of the Universe, even if it is not directly coupled to the Standard Model, through the exchange of a massive mediator. Indeed, thermal gravitino production $[11,15,16]$ was an early example of this type of process. In $[18,19,21-25]$ this type of dark matter production has been extended to Chern-Simons type couplings, spin-2 mediators, supergravity, and moduliportal scenarios respectively taking into account the effects of noninstantaneous reheating we discussed above $[27,28]$.

We can distinguish between the annihilation production processes and the decay rate by defining

$$
R(T)=R_{\text {annihilation }}(T)+R_{\text {decay }}(T) .
$$

We parametrize the production rate 


$$
R_{\text {annihilation }}(T)=\frac{T^{n+6}}{\Lambda^{n+2}},
$$

where $\Lambda$ is some beyond the Standard Model mass scale. ${ }^{3}$ If one looks at specific models, $n=2$ could correspond to the exchange of a massive particle with mass $\Lambda>T_{\max }$ or two nonrenormalizable mass suppressed couplings. Indeed, the $n=2$ case with a massive boson exchange has been discussed in Ref. [27], while it is also shown that single gravitino production in weak scale supersymmetry corresponds to $n=0 . n=6$ appears in processes invoking two mass suppression couplings and the exchange of a massive particle, which is the case in high scale supergravity [21-24] or moduli-portal scenarios [25]. In Sec. IV, we will discuss the $n=6$ case in a specific model where a pair of heavy gravitinos are produced by SM particle annihilation. The $n=10$ case can be realized in a non-Abelian dark matter model discussed in Ref. [18].

Inserting Eq. (11) in Eq. (8) we obtain, after integration, from $T_{\max }$ down to $T_{\mathrm{RH}}$, for the relic abundance at $T=T_{R H}:^{4}$

$$
\begin{aligned}
& \mathrm{n}<6: n\left(T_{R H}\right)=\frac{24 c}{5} \frac{T_{R H}^{n+4} M_{P}}{(6-n) \sqrt{3 \alpha} \Lambda^{n+2}} \\
& \mathrm{n}=6: n\left(T_{R H}\right)=\frac{24 c}{5 \sqrt{3 \alpha}} \frac{M_{P} T_{R H}^{10}}{\Lambda^{8}} \ln \left(\frac{T_{\max }}{T_{R H}}\right) \\
& \mathrm{n}>6: n\left(T_{R H}\right)=\frac{24 c}{5} \frac{T_{\max }^{n-6} M_{P} T_{R H}^{10}}{(n-6) \sqrt{3 \alpha} \Lambda^{n+2}}
\end{aligned}
$$

from which we can deduce the present relic abundance at the temperature $T_{0}$

$$
\begin{aligned}
\Omega & =\frac{\rho}{\rho_{c}} \approx \frac{n\left(T_{0}\right) \times m_{\chi}}{10^{-5} h^{2} \mathrm{GeV} \mathrm{cm}^{-3}} \\
& \Rightarrow \Omega h^{2} \approx 10^{5} \frac{n\left(T_{R H}\right)}{\mathrm{cm}^{-3}}\left(\frac{g\left(T_{0}\right)}{g\left(T_{R H}\right)}\right)\left(\frac{T_{0}}{T_{R H}}\right)^{3}\left(\frac{m_{\chi}}{1 \mathrm{GeV}}\right) \\
& \simeq 5.9 \times 10^{6}\left[\frac{n\left(T_{R H}\right)}{T_{R H}^{3}}\right]\left(\frac{m_{\chi}}{1 \mathrm{GeV}}\right)
\end{aligned}
$$

with $g(T)$ accounting for the relativistic degrees of freedom, where we have considered only Standard Model degrees of freedom $\left(g\left(T_{R H}\right)=106.75\right.$ and $\left.g\left(T_{0}\right)=3.91\right)$.

\footnotetext{
${ }^{3}$ This parametrization corresponds to a cross section $\sigma \propto \frac{T^{n}}{\Lambda^{n+2}}$.

${ }^{4}$ These expressions agree (using $c=1$ ) with the more exact derivation in [27] with the substitution of $\Lambda^{n+2}=$ $(\pi / \lambda)\left(\pi^{2} / g_{\chi} \zeta(3)\right)^{2} M^{n+2}$ where $g_{\chi}$ accounts for the internal number of degrees of freedom of the dark matter $\chi$. We note that the cross section was assumed to be $\sigma v=\lambda T^{n} / \pi M^{n+2}$ yielding a rate $R=n_{\chi}^{2} \sigma v$, with $n_{\chi}=g_{\chi} \zeta(3) T^{3} / \pi^{2}$.
}

\section{Dark matter production from inflaton decay}

We can also compute the dark matter density produced directly from the decay of the inflaton. Indeed, in Eq. (12), we neglected any direct couplings of the dark matter to the inflaton. But we can easily compute the relic abundance obtained if we allow a branching fraction $B_{R}$ of the inflaton decay into dark matter.

We will assume noninstantaneous thermalization and solve the Boltzmann equation Eq. (8) defining the production rate (number of dark matter particles produced per unit of time and volume) as

$$
R_{\text {decay }}(T)=\Gamma_{\phi} \frac{\rho_{\phi}}{M_{\phi}} N B_{R}=\frac{25}{12} \frac{\alpha^{2} T^{8}}{\Gamma_{\phi} M_{P}^{2} M_{\phi}} N B_{R},
$$

where $N$ is the number of dark matter quanta produced per inflaton decay. Plugging the rate Eq. (14) into Eq. (8), we obtain

$$
\frac{d Y}{d T}=-\frac{20}{3} \frac{\alpha}{M_{\phi} T^{5}} N B_{R},
$$

which gives after integration between $T_{\max }$ and $T_{\mathrm{RH}}$,

$$
\begin{aligned}
Y\left(T_{R H}\right) & \simeq \frac{5}{3} \frac{\alpha}{M_{\phi} T_{R H}^{4}} N B_{R}=\frac{g\left(T_{R H}\right) \pi^{2}}{18 M_{\phi} T_{R H}^{4}} N B_{R} \\
& \Rightarrow n\left(T_{R H}\right)=\frac{g\left(T_{R H}\right) \pi^{2}}{18 M_{\phi}} T_{R H}^{4} N B_{R} .
\end{aligned}
$$

Interestingly, this approximate result is remarkably close to the exact integration done in [30] where the coefficient of $\alpha T_{\mathrm{RH}}^{4} N B_{R} / M_{\phi}$ in $n$ is $(8 / 3)(c / 3)^{1 / 2}$ which is almost exactly $5 / 3$ when $c=1.2 .^{5}$

We can then combine Eq. (13) with Eq. (16) to obtain

$$
\begin{aligned}
\left.\Omega h^{2}\right|_{\text {decay }}= & \left(\frac{N B_{R}}{2.9 \times 10^{-9}}\right)\left(\frac{T_{R H}}{M_{\phi}}\right)\left(\frac{m}{1 \mathrm{GeV}}\right) \\
\simeq & 0.1\left(\frac{N B_{R}}{8.7 \times 10^{-7}}\right)\left(\frac{3 \times 10^{13} \mathrm{GeV}}{M_{\phi}}\right) \\
& \times\left(\frac{T_{R H}}{10^{10} \mathrm{GeV}}\right)\left(\frac{m}{1 \mathrm{GeV}}\right) .
\end{aligned}
$$

As we indicated earlier, we observe that one needs a very low branching ratio to avoid the overabundance of the dark matter. The total relic abundance is then obtained by adding the annihilation and decay contributions. Combining Eqs. (12) and (17), we obtain

$$
\left.\left.\Omega h^{2}\right|_{\text {tot }} \simeq \Omega h^{2}\right|_{\text {annihilation }}+\left.\Omega h^{2}\right|_{\text {decay }}
$$

\footnotetext{
${ }^{5}$ Notice that if we considered instantaneous reheating, one would have $n\left(T_{R H}\right)=N B_{R} \times \frac{\rho_{\phi}}{M_{\phi}}=N B_{R} \times \frac{g\left(T_{R H}\right) \pi^{2}}{30 M_{\phi}} T_{R H}^{4}$.
} 
or

$$
\begin{aligned}
& \left.\Omega h^{2}\right|_{n<6} \simeq 0.1\left[\frac{2.8 \times 10^{8} c}{(6-n)} \frac{T_{R H}^{n+1} M_{P}}{\sqrt{3 \alpha} \Lambda^{n+2}}+\frac{N B_{R}}{2.9 \times 10^{-10}} \times \frac{T_{R H}}{M_{\phi}}\right]\left(\frac{m}{1 \mathrm{GeV}}\right) \\
& \left.\Omega h^{2}\right|_{n=6} \simeq 0.1\left[2.8 \times 10^{8} c \frac{T_{R H}^{7} M_{P}}{\sqrt{3 \alpha} \Lambda^{8}} \ln \left(\frac{T_{\max }}{T_{R H}}\right)+\frac{N B_{R}}{2.9 \times 10^{-10}} \times \frac{T_{R H}}{M_{\phi}}\right]\left(\frac{m}{1 \mathrm{GeV}}\right) \\
& \left.\Omega h^{2}\right|_{n>6} \simeq 0.1\left[\frac{2.8 \times 10^{8} c}{(n-6)} \frac{T_{R H}^{7} T_{\max }^{n-6} M_{P}}{\sqrt{3 \alpha} \Lambda^{n+2}}+\frac{N B_{R}}{2.9 \times 10^{-10}} \times \frac{T_{R H}}{M_{\phi}}\right]\left(\frac{m}{1 \mathrm{GeV}}\right)
\end{aligned}
$$

which gives, for $n=6$ for example

$\left.\Omega h^{2}\right|_{n=6} ^{\text {tot }} \simeq 0.1\left[\left(\frac{T_{\mathrm{RH}}}{10^{11} \mathrm{GeV}}\right)^{7}\left(\frac{7.3 \times 10^{12} \mathrm{GeV}}{\Lambda}\right)^{8} \ln \left(\frac{T_{\mathrm{max}}}{T_{\mathrm{RH}}}\right)+\left(\frac{N B_{R}}{8.7 \times 10^{-8}}\right)\left(\frac{3 \times 10^{13} \mathrm{GeV}}{M_{\phi}}\right)\left(\frac{T_{\mathrm{RH}}}{10^{11} \mathrm{GeV}}\right)\right]\left(\frac{m}{1 \mathrm{GeV}}\right)$.

We show in Fig. 1 the result on the scan of the parameter space (in $N B_{R}, \Lambda$, and the dark matter mass, $m$ ) in the case $n=6$ using the relic density constraint $\Omega h^{2} \simeq 0.11$, for $T_{R H}=10^{11} \mathrm{GeV}$ and $T_{\max }=10^{13} \mathrm{GeV}$. For each value of $\Lambda, N B_{R}$, the mass needed to obtain the correct relic density is color coded by the scale at the right of the figure. We notice that the range of dark matter masses allowed is very large (from the $\mathrm{MeV}$ to the $\mathrm{EeV}$ scale). This is a direct consequence of the strong power dependence of the relic abundance on the scale $\Lambda$. We also remark that a branching ratio of $\mathcal{O}(1)$ is possible for dark matter masses of order $100 \mathrm{keV}$, whereas $\mathrm{EeV}$ scale dark matter requires very tiny

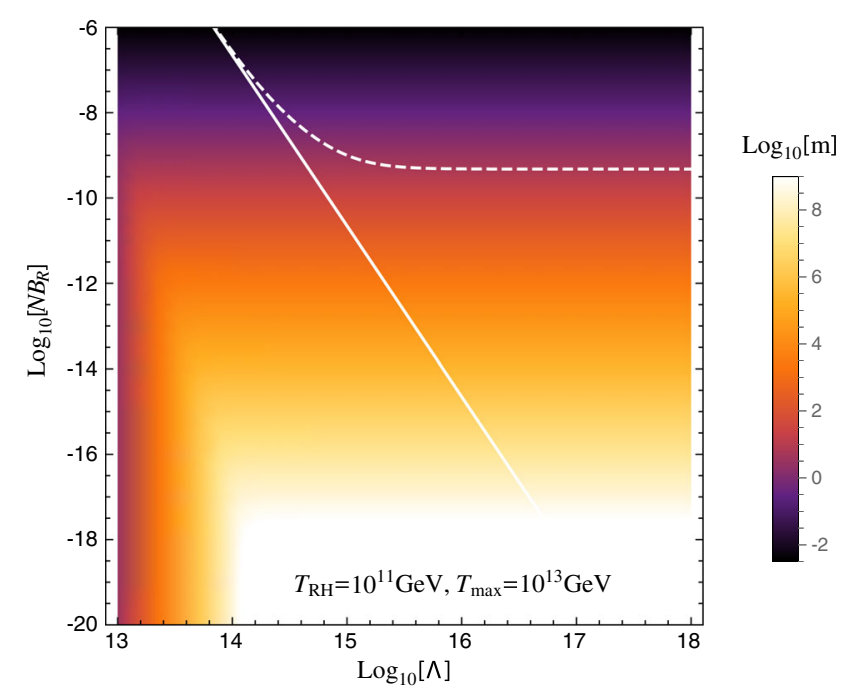

FIG. 1. Parameter space in the plane $\left(\Lambda, N B_{R}\right)$ of points respecting PLANCK constraints, with the corresponding dark matter mass $m$ for the case with $n=6$. The lines correspond to the branching ratio determined in our microscopic approach for $y_{\chi}=0$ (white solid line) and $y_{\chi}=10^{-4} y_{f}$ (white dashed line). See the text and Eq. (23) for details. branching fraction of the order of $N B_{R} \simeq 10^{-16}$ to avoid the overclosure of the Universe. Note also, that over most of the parameter space with large values of $\Lambda$, the thermal production from annihilations contributes negligibly to the relic density.

\section{GENERIC MICROSCOPIC MODELS}

We next apply the generic analysis we did using an effective field theory formulation. We first consider the effective Lagrangian ${ }^{6}$ between the inflaton $\phi$, Standard Model fields, $f$, and the dark matter candidate, $\chi$ :

$$
\mathcal{L}=y_{f} \phi \bar{f} f+y_{\chi} \phi \bar{\chi} \chi+\frac{1}{\Lambda^{2}} \bar{f} f \bar{\chi} \chi .
$$

This potential can be viewed as an effective interaction between Standard Model fermions and the dark sector through the exchange of a massive field of mass $M \simeq \Lambda \gg T_{\max }$.

One of our key points is that there will be direct production of dark matter through inflaton decay, even if the dark matter does not couple to the inflaton at tree level. That is, even if $y_{\chi}=0$, dark matter will be produced radiative via the diagram shown in Fig. 2. Indeed, from Eq. (20) one can deduce

$$
\Gamma_{\phi}^{\text {loop }} \simeq N_{f} \frac{y_{f} y_{\chi}}{16 \pi^{3}} \frac{M_{\phi}^{3}}{\Lambda^{2}}+N_{f} \frac{y_{f}^{2}}{128 \pi^{5}}\left(1+\frac{\pi^{2}}{4}\right) \frac{M_{\phi}^{5}}{\Lambda^{4}}
$$

via the loop of the $N_{f}$ families of the Standard Model fermions $f$, where the first term is the interference between

\footnotetext{
${ }^{6}$ Without loss of generalities, we will work with a fermionic dark matter candidate. The extension to a scalar candidate is straightforward.
} 


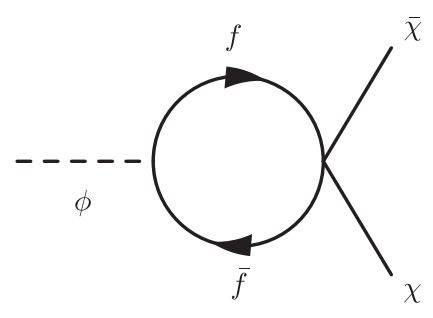

FIG. 2. The radiative decay of the inflaton into a pair of dark matter fields.

the tree and loop diagrams, and we have taken the massless limit for $f$. The other contributions to the inflaton width $\Gamma_{\phi}$ are given by

$$
\Gamma_{\phi}^{f}=\frac{N_{f} y_{f}^{2}}{8 \pi} M_{\phi} ; \quad \Gamma_{\phi}^{\chi}=\frac{y_{\chi}^{2}}{8 \pi} M_{\phi}
$$

We can express $B_{R}$ as function of the microscopic parameters,

$$
\begin{aligned}
B_{R}= & \frac{\Gamma_{\phi}^{\chi}+\Gamma_{\phi}^{\text {loop }}}{\Gamma_{\phi}^{f}+\Gamma_{\phi}^{\chi}+\Gamma_{\phi}^{\text {loop }}} \\
\simeq & \frac{1}{N_{f}}\left(\frac{y_{\chi}}{y_{f}}\right)^{2}+\frac{1}{2 \pi^{2}} \frac{y_{\chi}}{y_{f}}\left(\frac{M_{\phi}}{\Lambda}\right)^{2} \\
& +\frac{1}{16 \pi^{4}}\left(1+\frac{\pi^{2}}{4}\right)\left(\frac{M_{\phi}}{\Lambda}\right)^{4}
\end{aligned}
$$

where we assumed that $N_{f} y_{f} \gg y_{\chi}$ and $\Lambda \gg M_{\phi}$. These conditions are necessary to avoid the overabundance of dark matter. Also plotted in Fig. 1 is the corresponding branching ratio in the plane $\left(\Lambda, N B_{R}\right)$ for two different values of $y_{\chi}\left(y_{\chi}=0\right.$ and $\left.y_{\chi}=10^{-4} y_{f}\right)$. First of all, we notice that the lines lie in the region where the decay of the inflaton is responsible for the total amount of dark matter. The thermal production does not contribute in this area. Second, the plateau seen by the dashed curve corresponds to the points where the dark matter is completely produced by the tree level decay, uniquely determined by $y_{\chi}$ and thus independent of $\Lambda$. Moreover, even if $y_{\chi}=10^{-4} y_{f}$, for low values of $\Lambda \lesssim 5 \times 10^{14} \mathrm{GeV}$, the loop contribution dominates over the tree-level production.

Before closing this section, we briefly comment on direct/indirect searches for such dark matter. As is the case for super-weakly interacting dark matter, the dark matter interactions with the SM particles are so weak that neither direct nor indirect searches are possible (as long as dark matter is not decaying). In particular, the interactions between $\chi$ and $f$ in the model under consideration are suppressed by either $y_{f} y_{\chi} / M_{\phi}^{2}$ or $1 / \Lambda^{2}$, which provide extremely tiny couplings and in the case of direct detection, would result in scattering cross section below the neutrino floor.

\section{SUPERGRAVITY}

We next consider a more concrete supergravity model where the inflaton decays to only a pair of Higgs bosons at tree level, while a pair of gravitinos are produced via the loops of Higgses and Higgsinos. As a specific application, we would like to reconsider the high scale supergravity model described in [22]. The model is based on no-scale supergravity [32]. The inflationary and supersymmetry breaking sector contains three chiral fields, $T, \phi$ driving inflation [33], and a Polonyi-like field, $z$ [34] whose superpotential we take

$$
W=\sqrt{3} m_{3 / 2}(z+\nu)
$$

with $\nu$ being a constant. The Polonyi field is assumed to be twisted and strongly stabilized [35-37] so that the Kähler potential takes the form

$$
K=-3 \ln \left(T+\bar{T}-\frac{1}{3} \sum_{i}\left|\phi_{i}\right|^{2}\right)+|z|^{2}-\frac{|z|^{4}}{\Lambda_{z}^{2}},
$$

where one of the $\phi_{i}$ is related to inflation, and the rest are matter fields. Choosing [38],

$$
W=\sqrt{3} M_{T} \phi(T-1 / 2),
$$

leads to Starobinsky-like inflation [39] and is consistent with Planck observations [1] when the inflaton mass, which we now designate as $M_{T} \simeq 3 \times 10^{13} \mathrm{GeV}$.

If $\phi$ in (26) is fixed so that $\langle\phi\rangle=0$, the inflaton is associated with $T$ and its coupling to Standard Model fields leads to reheating, where the canonically normalized inflaton field $t$ is defined as $T \simeq(1 / 2)(1+\sqrt{2 / 3} t)$ about the minimum of $\langle T\rangle=1 / 2$. In a high scale supersymmetry model, all of the superpartners, except the gravitino, are assumed to be more massive than the inflaton. In particular, the $\mu$-parameter in the minimal supersymmetric SM is also large, $\mu>M_{T}$. In this case, the dominant decay mode for the inflaton is $t \rightarrow H_{u, d} H^{* u, d}$ which ultimately corresponds to a decay of $t \rightarrow h h$ where $h$ is the SM Higgs boson. The decay rate to two Higgs bosons is [40]

$$
\Gamma_{2 h}=\frac{\mu^{4}}{48 \pi M_{T} M_{P}^{2}} \equiv \frac{y^{2}}{8 \pi} M_{T}
$$

with $y^{2} \equiv \mu^{4} /\left(6 m_{T}^{2} M_{P}^{2}\right)$. Then the reheating temperature can be expressed as $T_{\mathrm{RH}} \simeq 0.5(y / 2 \pi) \sqrt{M_{T} M_{P}}[21,30,31]$ and $T_{\max } \simeq 0.5\left(8 \pi / y^{2}\right)^{1 / 4} T_{\mathrm{RH}}$.

Gravitinos are produced by the pair annihilation of SM particles, SM $\rightarrow$ two gravitinos, where the resultant gravitino abundance depends on the reheating process as discussed above. The reaction rate of the gravitino production is given by [20] 


$$
R \simeq \frac{21.65}{9} \frac{T^{12}}{m_{3 / 2}^{4} M_{\mathrm{P}}^{4}} \equiv \frac{T^{12}}{\Lambda^{8}}
$$

with $\Lambda^{8} \equiv 9 m_{3 / 2}^{4} M_{\mathrm{P}}^{4} / 21.65$. Then, from Eqs. (12) and (13), we obtain

$$
\begin{aligned}
\left.\Omega h^{2}\right|_{\text {ann }} ^{\text {inst }}= & 1.9 \times 10^{25} \frac{T_{R H}^{7}}{m_{3 / 2}^{3} M_{P}^{4}} \ln \left[\frac{T_{\text {max }}}{T_{\mathrm{RH}}}\right] \\
\simeq & 0.12\left(\frac{0.1 \mathrm{EeV}}{m_{3 / 2}}\right)^{3}\left(\frac{y}{2.3 \times 10^{-5}}\right)^{7} \\
& \times\left(\frac{M_{T}}{3 \times 10^{13} \mathrm{GeV}}\right)^{7 / 2} \ln \left(1.1 / y^{1 / 2}\right),
\end{aligned}
$$

where the instantaneous thermalization of SM particles is implicitly assumed. On the other hand, when the noninstantaneous thermalization effect in the gravitino production is incorporated, we have [28]

$$
\begin{aligned}
\left.\Omega h^{2}\right|_{\text {ann }} ^{\text {non-inst }}= & 0.12\left(\frac{0.1 \mathrm{EeV}}{m_{3 / 2}}\right)^{3}\left(\frac{M_{T}}{3 \times 10^{13} \mathrm{GeV}}\right)^{67 / 10} \\
& \times\left(\frac{y}{3.0 \times 10^{-7}}\right)^{19 / 5}\left(\frac{0.03}{\alpha_{3}}\right)^{16 / 5}
\end{aligned}
$$

where only gluon pair annihilation was assumed. We evaluate the $\mathrm{SU}(3)_{C}$ gauge coupling $\alpha_{3}=g_{s}^{2} / 4 \pi$ at $T_{R H}$ by solving renormalization group equations at twoloop level.

In this model, gravitinos are also produced by the treelevel decay of inflaton. Although the tree level coupling between gravitino and inflaton vanishes when $\langle\phi\rangle=0$, supersymmetry breaking shifts $\langle\phi\rangle$, giving rise to the treelevel decay given $b^{7}$

$$
\Gamma_{t}^{\text {tree }}=\left(\frac{\Lambda_{z}}{M_{P}}\right)^{4} \frac{81 m_{3 / 2}^{2} M_{T}}{128 \pi M_{P}^{2}}
$$

The radiative decay of inflaton to a pair of gravitinos is induced by the interactions given by

$$
\mathcal{L} \supset C_{t S S} t\left(\left|H_{u}\right|^{2}+\left|H_{d}\right|^{2}\right)+C_{t F F} t\left(\overline{\tilde{H}_{u}} \tilde{H}_{d}+\text { H.c. }\right),
$$

where $C_{t S S} \equiv \sqrt{2 / 3} \mu^{2}$ and $C_{t F F} \equiv \mu^{2} / 2 \sqrt{6}$ [40]. ${ }^{8}$ The other relevant terms in the supergravity Lagrangian are given by

\footnotetext{
${ }^{7}$ By comparing to the result of Ref. [40], the given expression is corrected by taking into account higher order terms of vacuum expectation values of $T, \phi$, and $z$, and the mixing between $t$ and $z$.

${ }^{8}$ Note that those interaction terms are obtained by using equations of motion. A more rigorous calculation would not use equations of motion when the interaction terms are relevant for loop diagrams. However, a more rigorous treatment of this particular process is not our main focus, and is expected to have only a small quantitative effect on our result.
}

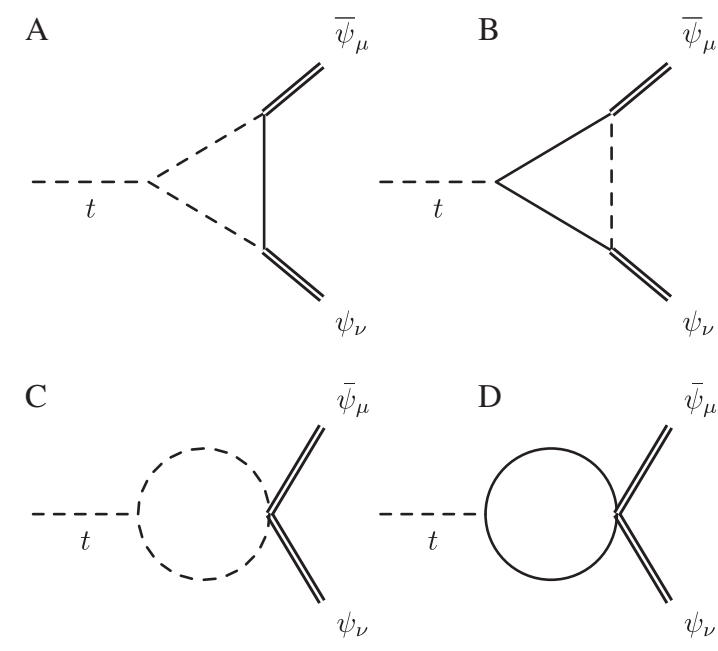

FIG. 3. The radiative decay of inflaton into a pair of gravitinos. Dashed (solid) lines in the loop represent the Higgs bosons (Higgsinos).

$$
\mathcal{L} \supset-\frac{i}{\sqrt{2} M_{P}}\left(\partial_{\mu} \varphi^{*}\right) \bar{\psi}_{\nu} \gamma^{\mu} \gamma^{\nu} \chi_{L}+\text { H.c. }
$$

where we denote a chiral multiplet $\left(\varphi, \chi_{L}\right)$. The relevant diagrams for the radiative decay of inflaton $t$ into a pair of gravitinos are shown in Fig. 3, where the dominant contribution is coming from the upper two diagrams (A and B). While a detailed discussion of the decay width is given in the Appendix, in the case of $m_{3 / 2} \ll M_{T} \ll \mu$ we obtain an approximate expression given by

$$
\Gamma^{\text {loop }} \simeq \frac{2}{3^{3} 4^{5} \pi^{5}}\left(\frac{1}{4}-\ln \frac{\mu^{2}}{\mu_{\text {ren }}^{2}}\right)^{2} \frac{\mu^{4} M_{T}^{5}}{m_{3 / 2}^{2} M_{P}^{6}},
$$

where $\mu_{\text {ren }}$ is the renormalization scale, and we take $\mu_{\text {ren }}=M_{T}$ in our analysis. Then, by using

$$
\begin{aligned}
& N B_{R} \simeq \frac{2\left(\Gamma^{\text {tree }}+\Gamma^{\text {loop }}\right)}{\Gamma_{2 h}} \equiv B_{R}^{\text {tree }}+B_{R}^{\text {loop }}, \\
& B_{R}^{\text {tree }}=\frac{243}{4}\left(\frac{\Lambda_{z}}{M_{P}}\right)^{4} \frac{m_{3 / 2}^{2} M_{T}^{2}}{\mu^{4}} \\
& \simeq 5.5 \times 10^{-12}\left(\frac{\Lambda_{z}}{M_{P}}\right)^{4}\left(\frac{m_{3 / 2}}{0.1 \mathrm{EeV}}\right)^{2} \\
& \times\left(\frac{M_{T}}{3 \times 10^{13} \mathrm{GeV}}\right)^{2}\left(\frac{10^{14} \mathrm{GeV}}{\mu}\right)^{4}, \\
& B_{R}^{\text {loop }} \simeq \frac{1}{144 \pi^{4}}\left(\frac{1}{4}-\ln \frac{\mu^{2}}{M_{T}^{2}}\right)^{2} \frac{M_{T}^{6}}{m_{3 / 2}^{2} M_{P}^{4}} \\
& \simeq 9.8 \times 10^{-15}\left(\frac{0.1 \mathrm{EeV}}{m_{3 / 2}}\right)^{2}\left(\frac{M_{T}}{3 \times 10^{13} \mathrm{GeV}}\right)^{6} \\
& \times\left[1-8 \ln \left(\frac{\mu}{M_{T}}\right)\right]^{2},
\end{aligned}
$$

we can evaluate $\left.\Omega h^{2}\right|_{\text {decay }}$ given in Eq. (17). 

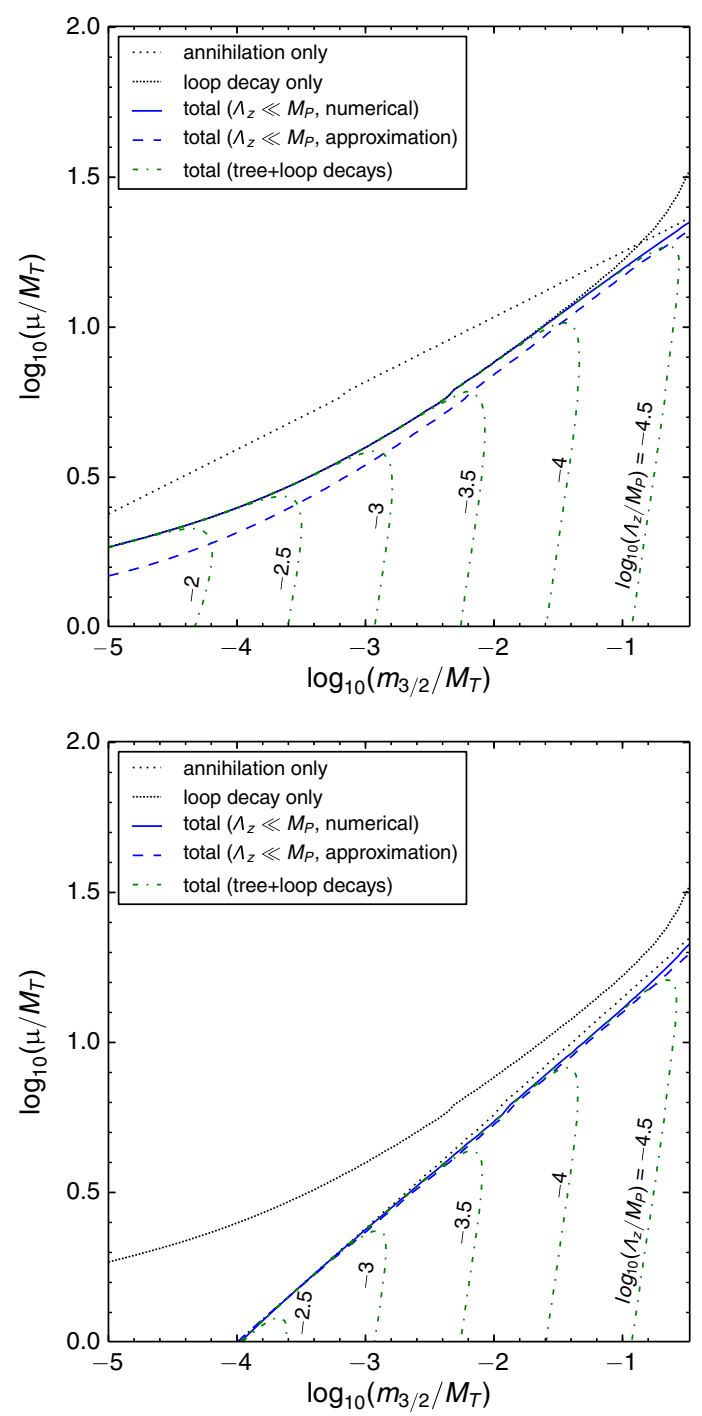

FIG. 4. The total number density of gravitino. The top panel assumes the instantaneous thermalization, and the bottom panel takes into account the effect of noninstantaneous thermalization.

Figure 4 shows the required relation between the $\mu$-parameter and the gravitino mass when total gravitino abundance given by $\left.\Omega h^{2}\right|_{\text {tot }}=\left.\Omega h^{2}\right|_{\text {ann }} ^{\text {inst } / \text { non-inst }}+$ $\left.\Omega h^{2}\right|_{\text {decay }}=0.12$, where we take $M_{T}=3 \times 10^{13} \mathrm{GeV}$. The top panel shows the case assuming instantaneous thermalization using Eq. (29), and the bottom panel takes the noninstantaneous thermalization effect into account using Eq. (30). In the both panels, the black dotted line shows the contribution from thermal production alone, using only $\left.\Omega h^{2}\right|_{\text {ann }}$. The short spaced dotted line shows the relation with only the loop contribution included. As one can see, the contribution from the 1-loop decay diagrams dominate over the thermal annihilation processes when $m_{3 / 2} \lesssim 10^{-1} M_{T}$. The solid blue contour corresponds the sum of the annihilation and loop decay contributions in
$\left.\Omega h^{2}\right|_{\text {tot }}=0.1197$ [1] evaluated without the approximation on the Passarino-Veltman functions [41], while the dashed blue line uses the approximate formula given in Eq. (34). To evaluate the Passarino-Veltman functions, we have utilized LoopTools [42]. For both blue lines, we take the tree-level decay contribution to be negligibly small by assuming $\Lambda_{z} \ll M_{P}$. The green dot-dashed lines are the total abundance with the tree level decay. The region below the solid blue line is the allowed parameter space.

\section{CONCLUSION}

The origin of the dark matter relic density is unknown. The thermal production mechanism [43], works quite well for weakly interacting massive particles. However, this mechanism does not work when the dark matter candidates are superweakly interacting and decouple very early (before inflation) in the history of the Universe. Gravitinos are a well known example of dark matter candidates for which their relic abundance is not determined by thermal annihilations. Instead, these particles are produced during the reheating process after inflation.

Some fraction of the dark matter may also be produced directly from inflaton decay if the inflaton couples to the dark matter candidate. However, even if the inflaton is not directly coupled to the dark matter, we have shown that direct production necessarily still occurs through radiative processes as in Fig. 2. We further showed in this work that direct production of dark matter through the radiative decay of the inflaton can in fact dominate the relic abundance in the Universe. We also note that in the generic model discussed in Sec. III, the predicted dark matter mass decreases for lower $\Lambda$ as shown in Fig. 1, which would be favorable to collider searches. However, the dark matter interactions with the SM particles are extremely weak, which indicates the lack of testability in current or near future experiments.

We highlighted this result with a specific example from no-scale supergravity. The model makes use of no-scale Starobinsky-like inflation, with supersymmetry breaking through a strongly stabilized Polonyi mechanism. We considered the case of high-scale supersymmetry in which all superpartners except the gravitino are more massive than the inflaton. Therefore, the thermal content of the universe is just that of the Standard Model. The dominant decay of the inflaton in this is model is to a pair of Standard Model Higgs bosons. The branching fraction of direct decays to gravitinos is suppressed [as given in Eq. (36)], though the degree of suppression depends on the strong stabilization parameter $\Lambda_{z}$. However the branching fraction to gravitinos through the loop process shown in Fig. 3 and given in Eq. (37) dominates over the thermal production when $m_{3 / 2}<10^{-1} M_{T} \sim 3 \times 10^{12} \mathrm{GeV}$. These heavy gravitinos may be detected through high energy cosmic ray neutrinos when R-parity is not conserved [23]. 


\section{ACKNOWLEDGMENTS}

The authors want to thank especially E. Dudas and M. Garcia for very insightful discussions. This research has been supported by the (Indo-French) CEFIPRA/IFCPAR Project No. 5404-2. Support from CNRS LIA-THEP and the INFRE-HEPNET of CEFIPRA/IFCPAR is also acknowledged. This work was also supported by the France-US Project International de Coopération Scientifique MicroDark. Y. M. acknowledges partial support from the European Union Horizon 2020 research and innovation programme under the Marie Sklodowska-Curie: RISE InvisiblesPlus (Grant Agreement No. 690575) and the International Training Network Elusives (Grant Agreement No. 674896). The work of K. K. and K. A. O. was supported in part by the DOE Grant No. DESC0011842 at the University of Minnesota.

\section{APPENDIX: CALCULATION OF THE RADIATIVE GRAVITINO DECAY}

The relevant gravitino interactions in the supergravity Lagrangian ${ }^{9}$ that involves Higgs and Higgsino are given by

$$
\begin{gathered}
\mathcal{L}_{3 \mathrm{pt}}=-\frac{i}{\sqrt{2}} \partial_{\mu} \varphi^{*} \bar{\psi}_{\nu} \gamma^{\mu} \gamma^{\nu} \chi_{L}+\frac{i}{\sqrt{2}} \partial_{\mu} \varphi \bar{\chi}_{L} \gamma^{\nu} \gamma^{\mu} \psi_{\nu}, \\
\mathcal{L}_{4 \mathrm{pt}}=\frac{1}{8} \epsilon^{\mu \nu \rho \sigma} \bar{\psi}_{\mu} \gamma_{\nu} \Phi_{\rho} \psi_{\sigma}-\frac{1}{4} e^{G / 2} \bar{\psi}_{\mu}\left[\gamma^{\mu}, \gamma^{\nu}\right] \psi_{\nu}, \\
\mathcal{L}_{4 \mathrm{~F}}=-\frac{1}{8} G_{i j^{*}} \bar{\psi}_{\mu}\left[i \epsilon^{\mu \nu \rho \sigma} \gamma_{\nu}+g^{\mu \rho} \gamma^{\sigma} \gamma^{5}\right] \psi_{\rho}\left(\bar{\chi}_{L}^{j} \gamma_{\sigma} \chi_{L}^{i}\right),
\end{gathered}
$$

where $G \equiv K+\ln |W|^{2}, G_{i}\left(G_{i j^{*}}\right)$ represents derivative of $G$ with respect to $\varphi^{i}\left(\varphi^{i}\right.$ and $\left.\varphi^{* j}\right)$, and $\Phi_{\rho} \equiv G_{i} \partial_{\rho} \varphi^{i}-$ $G_{i^{*}} \partial_{\rho} \varphi^{* i}$ which vanishes for $i=H_{u}$ and $H_{d}$. We also have $G_{H_{u} H_{u}^{*}}=G_{H_{d} H_{d}^{*}} \simeq 1$. The second term of Eq. (A2) gives Higgs-Higgs-gravitino-gravitino coupling. In the case of $m_{3 / 2} \ll \mu$, the dominant terms are induced by

$$
\left\langle e^{G / 2}\right\rangle \supset \frac{1}{2} \mu\left(H_{u} H_{d}+H_{u}^{\dagger} H_{d}^{\dagger}\right) .
$$

Thus, we have the four diagrams, shown in Fig. 3, that induce the radiative inflaton decay into a pair of gravitinos.

In calculating the decay amplitudes, the gravitino equation of motion (EOM) may be used to simplify the expressions, which is given as

$$
\epsilon^{\mu \nu \rho \sigma} \gamma^{5} \gamma_{\nu} \partial_{\rho} \psi_{\sigma}+\frac{1}{2} m_{3 / 2}\left[\gamma^{\mu}, \gamma^{\nu}\right] \psi_{\nu}=0 .
$$

Using the EOM, we also obtain the following relations:

\footnotetext{
${ }^{9}$ See, for instance, Ref. [44].
}

$$
\begin{gathered}
\left(\not p-m_{3 / 2}\right) \psi_{\mu}(p)=0, \\
\gamma^{\mu} \psi_{\mu}(p)=0, \\
p^{\mu} \psi_{\mu}(p)=0 .
\end{gathered}
$$

In the following discussion, we will use these equations to simplify the amplitudes whenever possible.

First, let us consider diagrams A and B. For a generic mass spectrum, the amplitudes are given by

$$
\begin{aligned}
i \hat{\mathcal{M}}_{A} & =\frac{1}{2} C_{t S S} \int \frac{d^{4} q}{(2 \pi)^{4}} \bar{u}_{\mu}(p) P_{L} \frac{\not \gamma^{\mu} \phi \gamma^{\nu} \phi}{D_{0} D_{1} D_{2}} v_{\nu}(k), \\
i \hat{\mathcal{M}}_{B}= & -\frac{1}{2} C_{t F F} \mu \int \frac{d^{4} q}{(2 \pi)^{4}} \bar{u}_{\mu}(p) P_{L}\left[2 \frac{\phi \gamma^{\mu} \phi \gamma^{\nu} \phi 1}{D_{0} D_{1} D_{2}}\right. \\
& \left.+\frac{\not \gamma^{\mu}\left(\not D_{1}+\not \phi_{2}\right) \gamma^{\nu} \not q}{D_{0} D_{1} D_{2}}\right] v_{\nu}(k),
\end{aligned}
$$

where we have omitted the factors arising from changing the basis of Higgs and Higgsinos into neutralinos and charginos, and the propagator in the denominators are defined as $D_{0}=q^{2}-m_{0}^{2}$ and $D_{i}=\left(q+p_{i}\right)^{2}-m_{i}^{2}(i=1,2)$. The gravitino wave functions are denoted as $u_{\mu}$ and $v_{\nu}$ which satisfy the Majorana condition $u_{\mu}=v_{\mu}^{c}$. The momenta in the propagators are taken to be $p_{1}=p$ and $p_{2}=-k$, while $m_{0}$ and $m_{1}\left(=m_{2}\right)$ are the Higgsino and Higgs boson masses for diagram A, and are the Higgs and Higgsino masses for diagram B, respectively. Then, the resultant amplitudes are given by

$$
\begin{aligned}
i \hat{\mathcal{M}}_{A}= & \frac{i C_{t S S}}{32 \pi^{2}}\left[C_{001}^{A}(\mathrm{I}+\mathrm{II}+\mathrm{III})+C_{002}^{A}(\mathrm{I}+\mathrm{II}+\mathrm{IV})\right. \\
& +C_{111}^{A} \mathrm{~V}+C_{222}^{A} \mathrm{VI} \\
& \left.+C_{112}^{A}(\mathrm{VII}+\mathrm{VIII}+\mathrm{IX})+C_{122}^{A}(\mathrm{X}+\mathrm{XI}+\mathrm{XII})\right] \\
= & \frac{i C_{t S S}}{32 \pi^{2}}\left[C_{001}^{A} \mathrm{III}+C_{002}^{A} \mathrm{IV}+C_{112}^{A} \mathrm{IX}+C_{122}^{A} \mathrm{X}\right],
\end{aligned}
$$

$$
\begin{aligned}
i \hat{\mathcal{M}}_{B}= & -2 i \hat{\mathcal{M}}_{A \leftrightarrow B} \\
& -\frac{i C_{t F F} \mu}{32 \pi^{2}}\left[C_{00}^{B}(\mathrm{III}+\mathrm{IV})+C_{11}^{B}(\mathrm{~V}+\mathrm{VIII})\right. \\
& \left.+C_{22}^{B}(\mathrm{XI}+\mathrm{VI})+C_{12}^{B}(\mathrm{VII}+\mathrm{IX}+\mathrm{X}+\mathrm{XII})\right] \\
= & -\frac{i C_{t F F} \mu}{32 \pi^{2}}\left[\left(C_{00}^{B}+2 C_{001}^{B}\right) \mathrm{III}+\left(C_{00}^{B}+2 C_{002}^{B}\right) \mathrm{IV}\right. \\
& \left.+\left(C_{12}^{B}+2 C_{112}^{B}\right) \mathrm{IX}+\left(C_{12}^{B}+2 C_{122}^{B}\right) \mathrm{X}\right],
\end{aligned}
$$

where $C_{i j}^{I}$ and $C_{i j k}^{I}$ are the Passarino-Veltman functions [41] for the diagram $I=A, B$, which are defined as 


$$
C_{\alpha} ; C_{\alpha \beta} ; C_{\alpha \beta \gamma}=\int \frac{d^{4} q}{i \pi^{2}} \frac{q_{\alpha} ; q_{\alpha} q_{\beta} ; q_{\alpha} q_{\beta} q_{\gamma}}{D_{0} D_{1} D_{2}}
$$

These functions may in general be written as

$$
\begin{gathered}
C_{\alpha}=p_{1 \alpha} C_{1}+p_{2 \alpha} C_{2}, \\
C_{\alpha \beta}=g_{\alpha \beta} C_{00}+p_{1 \alpha} p_{1 \beta} C_{11}+p_{2 \alpha} p_{2 \beta} C_{22} \\
+\left\{p_{1 \alpha} p_{2 \beta}+p_{2 \alpha} p_{1 \beta}\right\} C_{12}, \\
C_{\alpha \beta \gamma}=\sum_{i=1,2}\left\{g_{\alpha \beta} p_{i \gamma}+g_{\beta \gamma} p_{i \alpha}+g_{\gamma \alpha} p_{i \beta}\right\} C_{00 i} \\
+\left\{p_{1 \alpha} p_{1 \beta} p_{2 \gamma}+p_{1 \alpha} p_{2 \beta} p_{1 \gamma}+p_{2 \alpha} p_{1 \beta} p_{1 \gamma}\right\} C_{112} \\
+\left\{p_{2 \alpha} p_{2 \beta} p_{1 \gamma}+p_{2 \alpha} p_{1 \beta} p_{2 \gamma}+p_{1 \alpha} p_{2 \beta} p_{2 \gamma}\right\} C_{122}
\end{gathered}
$$

The terms denoted by I, II, III, ... are the amplitudes simplified by the EOM and the relevant relations, and are given by

(I) $\bar{u}_{\mu} P_{L} \gamma^{\alpha} \gamma^{\mu} \gamma_{\alpha} \gamma^{\nu} \not p_{i} v_{\nu}=0$,

(II) $\bar{u}_{\mu} P_{L} \not \not_{i} \gamma^{\mu} \gamma^{\beta} \gamma^{\nu} \gamma_{\beta} v_{\nu}=0$,

(III) $\bar{u}_{\mu} P_{L} \gamma^{\alpha} \gamma^{\mu} \not p_{1} \gamma^{\nu} \gamma_{\alpha} u_{\nu}=4 m_{3 / 2} g^{\mu \nu} \bar{u}_{\mu} P_{R} v_{\nu}$,

(IV) $\bar{u}_{\mu} P_{L} \gamma^{\alpha} \gamma^{\mu} \not p_{2} \gamma^{\nu} \gamma_{\alpha} u_{\nu}=4 m_{3 / 2} g^{\mu \nu} \bar{u}_{\mu} P_{L} v_{\nu}$,

(V) $\bar{v}_{\mu} P_{L} \not p_{1} \gamma^{\mu} \not p_{1} \gamma^{\nu} \not p_{1} v_{\nu}=0$

(VI) $\bar{u}_{\mu} P_{L} \not p_{2} \gamma^{\mu} \not p_{2} \gamma^{\nu} \not p_{2} v_{\nu}=0$

(VII) $\bar{u}_{\mu} P_{L} \not p_{1} \gamma^{\mu} \not \not 1_{1} \gamma^{\nu} \not \not_{2} v_{\nu}=0$

(VIII) $\bar{u}_{\mu} P_{L} \not p_{1} \gamma^{\mu} \not p_{2} \gamma^{\nu} \not \not_{2} v_{\nu}=0$

(IX) $\bar{u}_{\mu} P_{L} \not p_{2} \gamma^{\mu} \not p_{1} \gamma^{\nu} \not p_{1} v_{\nu}=-4 m_{3 / 2} k^{\mu} p^{\nu} \bar{u}_{\mu} P_{R} v_{\nu}$,

(X) $\bar{u}_{\mu} P_{L} \not p_{2} \gamma^{\mu} \not p_{2} \gamma^{\nu} \not p_{1} v_{\nu}=-4 m_{3 / 2} k^{\mu} p^{\nu} \bar{u}_{\mu} P_{L} v_{\nu}$,

(XI) $\bar{u}_{\mu} P_{L} \not \not_{2} \gamma^{\mu} \not \not p_{1} \gamma^{\nu} \not p_{2} v_{\nu}=0$

(XII) $\bar{u}_{\mu} P_{L} \not p_{1} \gamma^{\mu} \not \not_{2} \gamma^{\nu} \not p_{2} v_{\nu}=0$

where the momentum for $\bar{u}_{\mu}$ and $v_{\nu}$ is assigned to be $\bar{u}_{\mu}(p)$ and $v_{\nu}(k)$.

For diagram $\mathrm{C}$, we have

$$
i \hat{\mathcal{M}}_{C}=\frac{1}{4} C_{t S S} c_{H} \mu \int \frac{d^{4} q}{(2 \pi)^{4}} \bar{u}_{\mu}(p) P_{L} \frac{\Gamma^{\mu \sigma}}{D_{0} D_{1}} v_{\sigma}(k),
$$

where $c_{H}$ represents a constant factor coming from the mixing of Higgs bosons, and the vertex factor is defined as

$$
\Gamma^{\mu \sigma}=-\frac{1}{2}\left[\gamma^{\mu}, \gamma^{\sigma}\right] \text {. }
$$

Using Eq. (A7), we obtain

$$
i \hat{\mathcal{M}}_{C}=\frac{1}{8} \frac{i}{16 \pi^{2}} C_{t S S} \mu c_{H} B_{0} g^{\mu \nu} \bar{u}_{\mu} v_{\nu}
$$

where $B_{0}$ is the two-point scalar function defined as

$$
B_{0} \equiv \int \frac{d^{4} q}{i \pi^{2}} \frac{1}{D_{0} D_{1}}
$$

The coupling $c_{H}$ is found by recalling the relation between $H_{u, d}$ and their mass eigenstates ${ }^{10}$ given as

$$
\begin{gathered}
H_{u}^{0}=\frac{1}{\sqrt{2}}\left(c_{\alpha} h^{0}+s_{\alpha} H^{0}+i s_{\beta} G^{0}+i c_{\beta} A\right), \\
H_{d}^{0}=\frac{1}{\sqrt{2}}\left(-s_{\alpha} h^{0}+c_{\alpha} H^{0}-i c_{\beta} G^{0}+i s_{\beta} A\right), \\
H_{u}^{+}=s_{\beta} G^{+}+c_{\beta} H^{+}, \\
H_{d}^{-*}=-c_{\beta} G^{+}+s_{\beta} H^{+}
\end{gathered}
$$

with $s_{x} \equiv \sin x$ and $c_{x} \equiv \cos x$. We find

$$
\begin{aligned}
c_{H} B_{0}= & -\frac{1}{2} s_{\alpha} c_{\alpha} B_{0}\left(h^{0}\right) ;+\frac{1}{2} s_{\alpha} c_{\alpha} B_{0}\left(H^{0}\right) ; \\
& +\frac{1}{2} s_{\beta} c_{\beta} B_{0}\left(G^{0}\right) ;-\frac{1}{2} s_{\beta} c_{\beta} B_{0}(A) ; \\
& -s_{\beta} c_{\beta} B_{0}\left(G^{ \pm}\right) ;+s_{\beta} c_{\beta} B_{0}\left(H^{ \pm}\right) .
\end{aligned}
$$

In the $m_{A} \gg m_{Z}$ limit and $\tan \beta \simeq 1$, we have $-s_{\alpha} c_{\alpha} \sim$ $s_{\beta} c_{\beta} \sim 1 / 2$. Therefore, in the supersymmetric limit, all of the scalar masses are degenerate in mass at $\mu$, and the sum over all contributions identically vanishes. In the case that $m_{H^{0}} \sim m_{A} \sim m_{H^{ \pm}} \sim \mu \gg m_{h^{0}}$ and $m_{G^{0}}=m_{G^{ \pm}}=0$, we have

$$
\sum c_{H} B_{0} \simeq \frac{m_{h^{0}}^{2}}{M_{T}^{2}} \ll 1,
$$

so the contribution from diagram $\mathrm{C}$ turns out to be negligible.

For diagram $\mathrm{D}$, the amplitude is given by

$$
\begin{aligned}
i \hat{\mathcal{M}}_{D}= & -\frac{1}{8} C_{t F F} \int \frac{d^{4} q}{(2 \pi)^{4}} \bar{u}_{\mu}(p) \Gamma^{\mu \nu \sigma} v_{\nu}(k) \\
& \times \frac{\operatorname{tr}\left[(\not 1+m)\left(\not q+\not \phi_{t}+m\right) \gamma_{\sigma} P_{L}\right]}{D_{0} D_{1}},
\end{aligned}
$$

where $p_{t}$ is the inflaton momentum, and

$$
\Gamma^{\mu \nu \sigma}=i \epsilon^{\mu \alpha \nu \sigma} \gamma_{\alpha}+g^{\mu \nu} \gamma^{\sigma} \gamma^{5}
$$

After computing the trace in the amplitude, the loop integral is

$$
\int \frac{d^{4} q}{(2 \pi)^{4}} \frac{2 q_{\sigma}+p_{t, \sigma}}{D_{0} D_{1}}=\frac{i}{16 \pi^{2}} p_{t, \sigma}\left(2 B_{1}+B_{0}\right),
$$

\footnotetext{
${ }^{10} \mathrm{We}$ denote the lightest neutral Higgs $h^{0}$, heavier neutral Higgs $H^{0}, C P$-odd Higgs $A$, charged Higgs $H^{ \pm}$, neutral Goldstone boson $G^{0}$, and charged Goldstone boson $G^{ \pm}$.
} 
where $B_{1}$ is defined as

$$
\int \frac{d^{4} q}{i \pi^{2}} \frac{q_{\mu}}{D_{0} D_{1}}=p_{1 \mu} B_{1} .
$$

The spinor piece in the amplitude then gives

$$
\bar{u}_{\mu}(p) \Gamma^{\mu \rho \sigma} p_{t, \sigma} v_{\rho}(k)=\bar{u}_{\mu}\left[i \epsilon^{\mu \nu \rho \sigma} \gamma_{\nu} p_{t, \rho}+g^{\mu \rho} \not p_{t} \gamma^{5}\right] v_{\rho}
$$

The first term may be written as

$$
-2 m_{3 / 2} g^{\mu \rho} \bar{u}_{\mu}(p) \gamma^{5} v_{\rho}(k),
$$

where we have used the EOM. For the second term we obtain

$$
+2 m_{3 / 2} g^{\mu \rho} \bar{u}_{\mu}(p) \gamma^{5} v_{\rho}(k)
$$

where we have used $\not k v_{\rho}(k)=-m_{3 / 2} v_{\rho}(k)$ and $\bar{u}_{\mu}(p) \not p=$ $m_{3 / 2} \bar{u}_{\mu}(p)$. Therefore, the amplitude vanishes for diagram $\mathrm{D}$.

Hence, the total amplitude, whose dominant contributions come from diagrams $\mathrm{A}$ and $\mathrm{B}$, can be written as

$$
\begin{aligned}
i \hat{\mathcal{M}}_{\mathrm{tot}}= & 2 \frac{i m_{3 / 2}}{8 \pi^{2}}\left[c_{1 R} \hat{\mathcal{M}}_{1 R}+c_{1 L} \hat{\mathcal{M}}_{1 L}\right. \\
& \left.+c_{2 R} \hat{\mathcal{M}}_{2 R}+c_{2 L} \hat{\mathcal{M}}_{2 L}\right]
\end{aligned}
$$

where the overall factor 2 comes from the exchange of final state gravitinos, and we define

$$
\begin{aligned}
& \mathrm{III}=4 m_{3 / 2} \hat{\mathcal{M}}_{1 R}, \\
& \mathrm{IV}=4 m_{3 / 2} \hat{\mathcal{M}}_{1 L}, \\
& \mathrm{IX}=4 m_{3 / 2} \hat{\mathcal{M}}_{2 R}, \\
& \mathrm{X}=4 m_{3 / 2} \hat{\mathcal{M}}_{2 L}
\end{aligned}
$$

with the coefficients being

$$
\begin{aligned}
& c_{1 R}=C_{t S S} C_{001}^{A}-\mu C_{t F F}\left(C_{00}^{B}+2 C_{001}^{B}\right), \\
& c_{1 L}=C_{t S S} C_{002}^{A}-\mu C_{t F F}\left(C_{00}^{B}+2 C_{002}^{B}\right), \\
& c_{2 R}=C_{t S S} C_{112}^{A}-\mu C_{t F F}\left(C_{12}^{B}+2 C_{112}^{B}\right), \\
& c_{2 L}=C_{t S S} C_{122}^{A}-\mu C_{t F F}\left(C_{12}^{B}+2 C_{122}^{B}\right) .
\end{aligned}
$$

By denoting $F_{i, j} \equiv \mathcal{M}_{i}^{*} \mathcal{M}_{j}$ with $i=1 R, 1 L, 2 R, 2 L$, we have

$$
\begin{gathered}
F_{1 R, 1 R}=\frac{1}{3} M_{T}^{2} \tau \\
F_{1 L, 1 L}=F_{1 R, 1 R} \\
F_{1 R, 1 L}=-\frac{1}{9} M_{T}^{2} \tau^{-2}(-2+\tau)\left(8-8 \tau+\tau^{2}\right), \\
F_{2 R, 2 R}=\frac{2}{9} M_{T}^{6} \tau^{-1}(-1+\tau)^{2}, \\
F_{2 L, 2 L}=F_{2 R, 2 R}, \\
F_{1 R, 2 R}=-\frac{2}{9} M_{T}^{6} \tau^{-2}(-2+\tau)(-1+\tau)^{2}, \\
M_{T}^{4} \tau^{-1}(-2+\tau)(-1+\tau), \\
F_{1 L, 2 L}=F_{1 R, 2 R}, \\
F_{1 R, 2 L}=\frac{1}{9} M_{T}^{4} \tau^{-2}\left(-8+16 \tau-9 \tau^{2}+\tau^{3}\right), \\
F_{1 L, 2 R}=F_{1 R, 2 L},
\end{gathered}
$$

where $\tau \equiv 4 m_{3 / 2}^{2} / M_{T}^{2}$.

Next, let us express all Higgs and Higgsinos as mass eigenstates. ${ }^{11}$ The amplitude for $\mathrm{A}$ and $\mathrm{B}$ is given as

$$
\begin{aligned}
\mathcal{M}_{I}= & \hat{\mathcal{M}}_{I}\left(\tilde{\chi}_{2}^{ \pm}, H^{ \pm}\right)+\hat{\mathcal{M}}_{I}\left(\tilde{\chi}_{2}^{ \pm}, G^{ \pm}\right) \\
& +\frac{1}{4} \sum_{i=3,4}\left[\hat{\mathcal{M}}_{I}\left(\tilde{\chi}_{i}^{0}, h^{0}\right)+\hat{\mathcal{M}}_{I}\left(\tilde{\chi}_{i}^{0}, H^{0}\right)\right. \\
& \left.+\hat{\mathcal{M}}_{I}\left(\tilde{\chi}_{i}^{0}, G^{0}\right)+\hat{\mathcal{M}}_{I}\left(\tilde{\chi}_{i}^{0}, A\right)\right]
\end{aligned}
$$

for $I=A, B$, and thus we have,

$$
\begin{aligned}
\mathcal{M}_{\mathrm{A}+\mathrm{B}}= & \hat{\mathcal{M}}_{\mathrm{A}+\mathrm{B}}\left(\tilde{\chi}_{2}^{ \pm}, H^{ \pm}\right)+\hat{\mathcal{M}}_{\mathrm{A}+\mathrm{B}}\left(\tilde{\chi}_{2}^{ \pm}, G^{ \pm}\right) \\
& +\frac{1}{4} \sum_{i=3,4}\left[\hat{\mathcal{M}}_{\mathrm{A}+\mathrm{B}}\left(\tilde{\chi}_{i}^{0}, h^{0}\right)+\hat{\mathcal{M}}_{\mathrm{A}+\mathrm{B}}\left(\tilde{\chi}_{i}^{0}, H^{0}\right)\right. \\
& \left.+\hat{\mathcal{M}}_{\mathrm{A}+\mathrm{B}}\left(\tilde{\chi}_{i}^{0}, G^{0}\right)+\hat{\mathcal{M}}_{\mathrm{A}+\mathrm{B}}\left(\tilde{\chi}_{i}^{0}, A\right)\right] .
\end{aligned}
$$

As a good approximation for high-scale supersymmetry, let us suppose that $m_{\tilde{\chi}_{2}^{ \pm}} \sim m_{\chi_{3,4}^{0}} \sim m_{H^{ \pm}} \sim m_{H^{0}} \sim m_{A} \sim \mu \gg m_{h^{0}}$. Then, we may write the total amplitude as

$$
\mathcal{M}_{\mathrm{tot}} \simeq 2 \mathcal{M}_{\mathrm{tot}}^{h}+2 \mathcal{M}_{\mathrm{tot}}^{H}
$$

where $\mathcal{M}_{\text {tot }}^{h} \equiv \hat{\mathcal{M}}_{A+B}\left(\tilde{\chi}_{i}^{0}, h^{0}\right)=\hat{\mathcal{M}}_{A+B}\left(\tilde{\chi}_{i}^{0}, G^{0}\right)=\hat{\mathcal{M}}_{A+B} \times$ $\left(\tilde{\chi}_{i}^{0}, G^{ \pm}\right)$and $\mathcal{M}_{\mathrm{tot}}^{H} \equiv \hat{\mathcal{M}}_{A+B}\left(\tilde{\chi}_{2}^{ \pm}, H^{ \pm}\right)=\hat{\mathcal{M}}_{A+B}\left(\tilde{\chi}_{i}^{0}, H^{0}\right)=$ $\hat{\mathcal{M}}_{A+B}\left(\tilde{\chi}_{i}^{0}, A\right)$.

\footnotetext{
${ }^{11}$ We denote the Higgsino-like chargino as $\tilde{\chi}_{2}^{ \pm}$, and $\tilde{\chi}_{3,4}^{0}$ are the Higgsino-like neutralinos.
} 
We can further simplify the amplitude by taking the limit $m_{3 / 2} \ll M_{T} \ll \mu$. In this limit, the leading amplitudes among $F_{i, j}$ are $F_{1 R, 1 L}, F_{2 R, 2 L}$, and $F_{1 R, 2 L}$ for $\tau \ll 1$, namely,

$$
\begin{aligned}
F_{1 R, 1 L} & \simeq \frac{16}{9} M_{T}^{2} \tau^{-2}, \\
F_{2 R, 2 L} & \simeq \frac{4}{9} M_{T}^{6} \tau^{-2}, \\
F_{1 R, 2 L} & \simeq-\frac{8}{9} M_{T}^{4} \tau^{-2} .
\end{aligned}
$$

Besides, as we can see from the dimensionality, $C_{00}$ and $C_{00 i}$ in $c_{1 R}$ and $c_{1 L}$ remain constant (or logarithmic of the involved mass scales, i.e., $\mu$ ) as it is dimensionless, while $C_{12}^{B}$ in $c_{2 R}$ and $c_{2 L}$ scales as $1 / \mu^{2}$. Indeed, we obtain analytic expressions of those functions in this limit:

$$
\begin{gathered}
C_{001}^{A, h} \simeq-\frac{1}{9}+\frac{1}{12} \ln \frac{\mu^{2}}{\mu_{\mathrm{ren}}^{2}}, \\
C_{001}^{A, H} \simeq \frac{1}{24}+\frac{1}{12} \ln \frac{\mu^{2}}{\mu_{\mathrm{ren}}^{2}}, \\
C_{00}^{B, h} \simeq-\frac{1}{4} \ln \frac{\mu^{2}}{\mu_{\mathrm{ren}}^{2}},
\end{gathered}
$$

$$
\begin{aligned}
& C_{00}^{B, H} \simeq-\frac{1}{8}-\frac{1}{4} \ln \frac{\mu^{2}}{\mu_{\mathrm{ren}}^{2}}, \\
& C_{001}^{B, h} \simeq \frac{1}{72}+\frac{1}{12} \ln \frac{\mu^{2}}{\mu_{\mathrm{ren}}^{2}}, \\
& C_{001}^{B, H} \simeq \frac{1}{24}+\frac{1}{12} \ln \frac{\mu^{2}}{\mu_{\mathrm{ren}}^{2}},
\end{aligned}
$$

and $C_{001}^{I, h(H)}=C_{002}^{I, h(H)}$ for $I=A, B$. Therefore, the dominant contribution in the decay width is the term involving $\operatorname{Re}\left(c_{1 R}^{*} c_{1 L}\right) F_{1 R, 1 L}$ in the squared amplitude. Then, we obtain

$$
\begin{aligned}
\Gamma_{\phi \rightarrow 3 / 2}^{\text {loop }} & =\frac{\left|\mathcal{M}_{\mathrm{tot}}\right|^{2}}{2 \cdot 16 \pi M_{T}} \simeq \frac{\operatorname{Re}\left(\bar{c}_{1 R}^{*} \bar{c}_{1 L}\right)}{3^{2} 4^{4} \pi^{5}} \frac{M_{T}^{5}}{m_{3 / 2}^{2}} \\
& \simeq \frac{2}{3^{3} 4^{5} \pi^{5}}\left(\frac{1}{4}-\ln \frac{\mu^{2}}{\mu_{\mathrm{ren}}^{2}}\right)^{2} \frac{\mu^{4} M_{T}^{5}}{m_{3 / 2}^{2} M_{P}^{6}},
\end{aligned}
$$

where $\bar{c}_{i} \equiv c_{i}^{h}+c_{i}^{H}$ for $i=1 R, 1 L, 2 R, 2 L$, and we have used

$$
\begin{aligned}
\bar{c}_{1 R} \simeq \bar{c}_{1 L} & \simeq-\frac{C_{t S S}}{2}\left[2 C_{00}^{B, h}+2 C_{00}^{B, H}\right] \\
& \simeq-\frac{1}{2} \sqrt{\frac{2}{3}} \mu^{2}\left(\frac{1}{4}-\ln \frac{\mu^{2}}{\mu_{\mathrm{ren}}^{2}}\right)
\end{aligned}
$$

with the relation $C_{t S S}=2 \mu C_{t F F}$.
[1] P. A. R. Ade et al. (Planck Collaboration), Astron. Astrophys. 594, A13 (2016); N. Aghanim et al. (Planck Collaboration), arXiv:1807.06209.

[2] E. Aprile et al. (XENON Collaboration), Phys. Rev. Lett. 119, 181301 (2017).

[3] D. S. Akerib et al. (LUX Collaboration), Phys. Rev. Lett. 118, 021303 (2017).

[4] X. Cui et al. (PandaX-II Collaboration), Phys. Rev. Lett. 119, 181302 (2017).

[5] V. Silveira and A. Zee, Phys. Lett. 161B, 136 (1985); J. McDonald, Phys. Rev. D 50, 3637 (1994); C. P. Burgess, M. Pospelov, and T. ter Veldhuis, Nucl. Phys. B619, 709 (2001); H. Davoudiasl, R. Kitano, T. Li, and H. Murayama, Phys. Lett. B 609, 117 (2005); H. Han and S. Zheng, J. High Energy Phys. 12 (2015) 044.

[6] J. A. Casas, D. G. Cerdeo, J. M. Moreno, and J. Quilis, J. High Energy Phys. 05 (2017) 036; A. Djouadi, O. Lebedev, Y. Mambrini, and J. Quevillon, Phys. Lett. B 709, 65 (2012); A. Djouadi, A. Falkowski, Y. Mambrini, and J. Quevillon, Eur. Phys. J. C 73, 2455 (2013); O. Lebedev, H. M. Lee, and Y. Mambrini, Phys. Lett. B 707, 570 (2012); Y. Mambrini, Phys. Rev. D 84, 115017 (2011).
[7] J. Ellis, A. Fowlie, L. Marzola, and M. Raidal, Phys. Rev. D 97, 115014 (2018); G. Arcadi, Y. Mambrini, and F. Richard, J. Cosmol. Astropart. Phys. 03 (2015) 018; J. Kearney, N. Orlofsky, and A. Pierce, Phys. Rev. D 95, 035020 (2017); M. Escudero, A. Berlin, D. Hooper, and M. X. Lin, J. Cosmol. Astropart. Phys. 12 (2016) 029.

[8] A. Alves, S. Profumo, and F. S. Queiroz, J. High Energy Phys. 04 (2014) 063; O. Lebedev and Y. Mambrini, Phys. Lett. B 734, 350 (2014); G. Arcadi, Y. Mambrini, M. H. G. Tytgat, and B. Zaldivar, J. High Energy Phys. 03 (2014) 134; O. Lebedev and Y. Mambrini, Phys. Lett. B 734, 350 (2014).

[9] H. P. Nilles, Phys. Rep. 110, 1 (1984); H. E. Haber and G. L. Kane, Phys. Rep. 117, 75 (1985).

[10] H. Goldberg, Phys. Rev. Lett. 50, 1419 (1983).

[11] J. Ellis, J. Hagelin, D. Nanopoulos, K. Olive, and M. Srednicki, Nucl. Phys. B238, 453 (1984).

[12] G. Arcadi, M. Dutra, P. Ghosh, M. Lindner, Y. Mambrini, M. Pierre, S. Profumo, and F. S. Queiroz, Eur. Phys. J. C 78, 203 (2018).

[13] L. J. Hall, K. Jedamzik, J. March-Russell, and S. M. West, J. High Energy Phys. 03 (2010) 080; X. Chu, T. Hambye, 
and M. H. G. Tytgat, J. Cosmol. Astropart. Phys. 05 (2012) 034; X. Chu, Y. Mambrini, J. Quevillon, and B. Zaldivar, J. Cosmol. Astropart. Phys. 01 (2014) 034; A. Biswas, D. Borah, and A. Dasgupta, Phys. Rev. D 99, 015033 (2019).

[14] N. Bernal, M. Heikinheimo, T. Tenkanen, K. Tuominen, and V. Vaskonen, Int. J. Mod. Phys. A 32, 1730023 (2017).

[15] D. V. Nanopoulos, K. A. Olive, and M. Srednicki, Phys. Lett. 127B, 30 (1983).

[16] M. Y. Khlopov and A. D. Linde, Phys. Lett. 138B, 265 (1984).

[17] Y. Mambrini, K. A. Olive, J. Quevillon, and B. Zaldivar, Phys. Rev. Lett. 110, 241306 (2013); Y. Mambrini, N. Nagata, K. A. Olive, J. Quevillon, and J. Zheng, Phys. Rev. D 91, 095010 (2015); N. Nagata, K. A. Olive, and J. Zheng, J. High Energy Phys. 10 (2015) 193; Y. Mambrini, N. Nagata, K. A. Olive, and J. Zheng, Phys. Rev. D 93, 111703 (2016); N. Nagata, K. A. Olive, and J. Zheng, J. Cosmol. Astropart. Phys. 02 (2017) 016; X. Chu, Y. Mambrini, J. Quevillon, and B. Zaldivar, J. Cosmol. Astropart. Phys. 01 (2014) 034.

[18] G. Bhattacharyya, M. Dutra, Y. Mambrini, and M. Pierre, Phys. Rev. D 98, 035038 (2018).

[19] N. Bernal, M. Dutra, Y. Mambrini, K. Olive, M. Peloso, and M. Pierre, Phys. Rev. D 97, 115020 (2018).

[20] K. Benakli, Y. Chen, E. Dudas, and Y. Mambrini, Phys. Rev. D 95, 095002 (2017).

[21] E. Dudas, Y. Mambrini, and K. Olive, Phys. Rev. Lett. 119, 051801 (2017).

[22] E. Dudas, T. Gherghetta, Y. Mambrini, and K. A. Olive, Phys. Rev. D 96, 115032 (2017).

[23] E. Dudas, T. Gherghetta, K. Kaneta, Y. Mambrini, and K. A. Olive, Phys. Rev. D 98, 015030 (2018).

[24] S. A. R. Ellis, T. Gherghetta, K. Kaneta, and K. A. Olive, Phys. Rev. D 98, 055009 (2018).

[25] D. Chowdhury, E. Dudas, M. Dutra, and Y. Mambrini, arXiv:1811.01947 [Phys. Rev. D (to be published)].

[26] G. F. Giudice, E. W. Kolb, and A. Riotto, Phys. Rev. D 64, 023508 (2001); D. J. H. Chung, E. W. Kolb, and A. Riotto, Phys. Rev. D 60, 063504 (1999).

[27] M. A. G. Garcia, Y. Mambrini, K. A. Olive, and M. Peloso, Phys. Rev. D 96, 103510 (2017).
[28] M. A. G. Garcia and M. A. Amin, Phys. Rev. D 98, 103504 (2018).

[29] P. S. Bhupal Dev, A. Mazumdar, and S. Qutub, Front. Phys. 2, 26 (2014).

[30] J. Ellis, M. A. G. Garcia, D. V. Nanopoulos, K. A. Olive, and M. Peloso, J. Cosmol. Astropart. Phys. 03 (2016) 008.

[31] J. Pradler and F. D. Steffen, Phys. Lett. B 648, 224 (2007).

[32] E. Cremmer, S. Ferrara, C. Kounnas, and D. V. Nanopoulos, Phys. Lett. 133B, 61 (1983); J. R. Ellis, A. B. Lahanas, D. V. Nanopoulos, and K. Tamvakis, Phys. Lett. 134B, 429 (1984).

[33] J. Ellis, D. V. Nanopoulos, and K. A. Olive, Phys. Rev. Lett. 111, 111301 (2013); R. Kallosh and A. Linde, J. Cosmol. Astropart. Phys. 06 (2013) 028; F. Farakos, A. Kehagias, and A. Riotto, Nucl. Phys. B876, 187 (2013); J. Ellis, D. V. Nanopoulos, and K. A. Olive, J. Cosmol. Astropart. Phys. 10 (2013) 009; J. Ellis, D. V. Nanopoulos, K. A. Olive, and S. Verner, arXiv:1812.02192.

[34] J. Polonyi, Hungary Central Research Institute Report No. KFKI-1977-93, 1977.

[35] M. Dine, R. Kitano, A. Morisse, and Y. Shirman, Phys. Rev. D 73, 123518 (2006); R. Kitano, Phys. Lett. B 641, 203 (2006).

[36] J. L. Evans, M. A. G. Garcia, and K. A. Olive, J. Cosmol. Astropart. Phys. 03 (2014) 022.

[37] E. Dudas, A. Linde, Y. Mambrini, A. Mustafayev, and K. A. Olive, Eur. Phys. J. C 73, 2268 (2013).

[38] S. Cecotti, Phys. Lett. B 190, 86 (1987).

[39] A. A. Starobinsky, Phys. Lett. 91B, 99 (1980).

[40] J. Ellis, M. A. G. Garcia, D. V. Nanopoulos, and K. A. Olive, J. Cosmol. Astropart. Phys. 10 (2015) 003.

[41] G. Passarino and M. J. G. Veltman, Nucl. Phys. B160, 151 (1979).

[42] T. Hahn and M. Perez-Victoria, Comput. Phys. Commun. 118, 153 (1999).

[43] P. Hut, Phys. Lett. 69B, 85 (1977); B. W. Lee and S. Weinberg, Phys. Rev. Lett. 39, 165 (1977).

[44] E. Cremmer, S. Ferrara, L. Girardello, and A. Van Proeyen, Nucl. Phys. B212, 413 (1983); J. Wess and J. Bagger, Supersymmetry and Supergravity (Princeton University Press, Princeton, NJ, 1992). 